\title{
A Transition State Theory-influenced Model of Secondary Battery Cycle-life: Towards a Final Regularity Resembling Carnot-efficiency
}

\author{
Roland H. Pawelke
}

\begin{abstract}
A transition state theory-influenced approach on maximum battery cycle-life is outlined, arriving at an ideal model of general validity. The outcome may be understood further as a thermodynamic final regularity reminiscent of Carnotefficiency. In contrast to the common perception which attributes in blanket fashion the causality of changes in cycle-life to the engineering of battery-specific tangibles, this model allows for a more differentiated picture: That changes to batteryspecific tangibles may yield differences of several hundred or more cycles is here the result of them being enhanced by a comparatively long, natural constant-based, logarithmic lever. That way such changes can cause big differences though being comparatively small to the lever base value, which emerges as a quantity of natural constants, temperature(s) and relative capacity margins but independent of battery specific energy and applied power. These are findings suggesting a revision of the current empirics-biased consensus opinion about the matter.
\end{abstract}

\section{Introduction}

How many charge-discharge cycles a secondary battery may deliver under operational conditions belongs to the prime questions of reversible electrochemical energy storage. Approaches towards modelling that quantity of interest exist in literature and are either of electrochemical-physical character or (semi-)empirical nature: ${ }^{1}$ Both reflect the empiric positivist bias seemingly ruling the matter of reversible chemical energy storage ever since and emphasize the predominance or even exclusive significance of system-specific tangibles. While that bias is accountable considering the drive towards products and applications much less so is the total absence of chemistry approaches based on molecular conceptions. That is because the reversible transfer of mass, the one central concept to reversible chemical energy storage, is located right at the ideal theoretical fundament of physical chemistry. Considering the vast practical significance of reversible chemical energy storage, it is not an excessive expectation that at least some ideal idea about the attainable specific energy and battery cycle-life could/should have materialized a long time ago. Yet the discrepancy from that expectation is complete and stretches to the present day.

How the fundamentals of physical chemistry set the limits to reversible specific energy has been already outlined by this author on previous occasions; ${ }^{2,3}$ yet pinning down by the selfsame principles how often a reversible reaction may ideally occur under failure conditions remained an open issue to date. This paper is set for closure on that matter and approaches the issue from a transition state theory (TST) angle as it inherently relates equilibrium thermodynamics to kinetics, thus accounting for an ambiguous role of time, which is a vital characteristic of the problem at hand.

\section{Methodical Approach}

Central to TST is the concept of a fast upstream equilibrium between the reactants and an activated complex in which the reaction products are virtually already present; the kinetically measurable reaction event is triggered by a molecular vibration along the reaction coordinate, causing product formation. ${ }^{4}$ This vibration is accounted for by means of a universal frequency factor $\mathrm{k}_{B} T / \mathrm{h}$ in which $\mathrm{k}_{\mathrm{B}}$ and $\mathrm{h}$ represent the Boltzmann and the Planck constant, respectively. Equation 1 shows the classic 
Eyring-Polanyi equation connecting the reaction rate $k$ to the molar standard Gibbs enthalpy $\Delta G_{\mathrm{m}}{ }^{\circ} \neq$ which governs the equilibrium between reactants and the activated complex.

$k=\frac{\mathrm{k}_{\mathrm{B}} T}{\mathrm{~h}} \mathrm{e}^{-\Delta G_{\mathrm{m}}{ }^{\circ} / \mathrm{R} T}$

The basic TST concept of an upstream equilibrium prior the actual reaction event may be applied in adjusted form to secondary batteries because of the fundamental equilibrium nature of reversible (electro-)chemical systems. Hence anything prior the most activated, thus slowest, thus kinetically visible step of the reaction may be subsumed in equilibrium manner and in a secondary battery the intercalation of the migrating species into the vacant electrode site qualifies as such a bottleneck step. Thus, the upstream equilibrium covers the mass transfer from one electrode just towards the vacant destination site in the other. The shift from a single-phase equilibrium towards a coupled multi-phase one is unproblematic as long as the adjustment of the global equilibrium occurs still faster than the intercalation of the migrating ion at the vacant site. A molecular vibration along the reaction coordinate triggers the apparent reaction event respective the charge or discharge process.

However, the present scope requires that said global equilibrium relation is augmented by a specific (i.e. mass) reference. This additional information provides the equilibrium constant with the quality of a reservoir of reaction events because a given amount of system mass can allow for only so many of them: in relation to equation 1 that may be achieved by expressing the chemical reaction rate $k$ via the ratio of specific battery power in $\mathrm{W} \mathrm{kg}^{-1}$ by battery specific energy in $\mathrm{Wh} \mathrm{kg}^{-1}$. How reaction event count relates to maximum cycle-life is as follows: in each cycle, the real system will experience capacity degradation and the minimum causal increment to that process is one molecular reaction event falling away per macroscopic cycle. The loss of one reaction event over two cycles does not make sense because failure is a binary matter in this context: on what grounds could the chemistry have worked in the first cycle but then failed in the subsequent one? There are none; in contrast, knowing about the actual reason why a reaction event failed is non-essential as it does not matter why the thermodynamic driving force became insufficient, it only matters that it did. By that line of argument it is assessed how often the Gibbs enthalpy of the reaction suffices to vault the system over a residual capacity bar while each time one reaction event breaks away: due to the causality of minimum degradation this gives the ideal cycle-life of a battery under real failure conditions.

Hence equation 1 is adapted towards equations 2 and 3: The mass transfer of the migrating species $M$ between the cathode $(C)$ and the anode $(A)$ occurs via two respective global upstream equilibria of the constants $K_{1}$ and $K_{2}$, each reaching from the occupied site by $M$ in one electrode upfront the vacant destination site in the other (indicated by dots). Both equilibria adjust faster than the incorporation of $\mathrm{M}$ into the target vacancy. The respective equilibrium constants are outfitted with an implicit specific reference via the kinetic reaction constants as sketched, creating the connotation of reaction event reservoirs. The reactions occur at two distinct temperatures $T_{1}$ and $T_{2}$. Overall, the respective apparent reaction rates $k_{1}$ and $k_{2}$ are defined in analogy to the classic Eyring-Polanyi equation but the index $\neq$ is omitted because the activated complex and its properties are of no concern to the actual scope. 


$$
\begin{array}{lll}
\mathrm{C} \cdot \mathrm{M} \rightleftharpoons[\mathrm{C} \cdots \mathrm{M} \cdot \mathrm{A}] \rightarrow \mathrm{A} \cdot \mathrm{M} & \Rightarrow & -\mathrm{R} T_{1} \ln K_{1}=\Delta G_{m, 1}{ }^{\circ}=-\mathrm{R} T_{1} \ln \left(\frac{k_{1} \mathrm{~h}}{\mathrm{k}_{\mathrm{B}} T_{1}}\right) \\
\mathrm{C} \cdot \mathrm{M} \leftarrow[\mathrm{C} \cdot \mathrm{M} \cdots \mathrm{A}] \rightleftharpoons \mathrm{A} \cdot \mathrm{M} & \Rightarrow & -\mathrm{R} T_{2} \ln K_{2}=\Delta G_{\mathrm{m}, 2^{\circ}}=-\mathrm{R} T_{2} \ln \left(\frac{k_{2} \mathrm{~h}}{\mathrm{k}_{\mathrm{B}} T_{2}}\right)
\end{array}
$$

\section{Results}

Central to this problem is its multi-layered structure: there is a macroscopic cycle and a microscopic reaction event level and bringing these soundly together is the task at hand. Equation 4 displays the base hypothesis of this approach and constructs by means of the sum of both Gibbs enthalpies via the relation $-\mathrm{R} T \ln K=\Delta G_{\mathrm{m}}{ }^{\circ}$ an equilibrium constant $K_{1,2}$ (a mere auxiliary quantity) which is a measure of total reaction event count per macroscopic cycle.

$$
-\left(\frac{\Delta G_{m, 1}{ }^{\circ}+\Delta G_{m, 2}{ }^{\circ}}{R}\right)=\ln K_{1,2}=\ln \left(\#_{\text {reaction events }}\right)
$$

Equation 4 is expressed in terms of equations 2 and 3 as shown in equation 5.

$$
-\left(\frac{\Delta G_{\mathrm{m}, 1}{ }^{\circ}+\Delta G_{\mathrm{m}, 2}{ }^{\circ}}{\mathrm{R}}\right)=T_{1} \ln \left(\frac{k_{1} \mathrm{~h}}{\mathrm{k}_{\mathrm{B}} T_{1}}\right)+T_{2} \ln \left(\frac{k_{2} \mathrm{~h}}{\mathrm{k}_{\mathrm{B}} T_{2}}\right)=\ln \left(\#_{\text {reaction events }}\right)
$$

Reversibility implies equal thermodynamic driving forces back and forth and due to that symmetry requirement both Gibbs enthalpies are equal but in order to unify indexes, both are expressed by the arithmetic mean $\Delta G_{\mathrm{m}, \mathrm{a}}{ }^{\circ}$ as equation 6 shows.

$$
-\left(\frac{\Delta G_{\mathrm{m}, 1}{ }^{\circ}+\Delta G_{\mathrm{m}, 2}{ }^{\circ}}{\mathrm{R}}\right)=-\left(\frac{2 \Delta G_{\mathrm{m}, \mathrm{a}}{ }^{\circ}}{\mathrm{R} T_{\mathrm{a}}}\right)=\left(T_{1} \ln \left(\frac{k_{1} \mathrm{~h}}{\mathrm{k}_{\mathrm{B}} T_{1}}\right)+T_{2} \ln \left(\frac{k_{2} \mathrm{~h}}{\mathrm{k}_{\mathrm{B}} T_{2}}\right)\right)
$$

The pre-logarithmic temperatures $T_{1}$ and $T_{2}$ are expressed in terms of their arithmetic mean $T_{\mathrm{a}}$ accordingly which is isolated and brought to the left side as equations $7 a$ to $7 c$ show.

$$
\begin{aligned}
& -\left(\frac{2 \Delta G_{\mathrm{m}, \mathrm{a}}^{\circ}}{\mathrm{R}}\right)=\left(\left(2 T_{\mathrm{a}}-T_{2}\right) \ln \left(\frac{k_{1} \mathrm{~h}}{\mathrm{k}_{\mathrm{B}} T_{1}}\right)+\left(2 T_{\mathrm{a}}-T_{1}\right) \ln \left(\frac{k_{2} \mathrm{~h}}{\mathrm{k}_{\mathrm{B}} T_{2}}\right)\right) \\
& -\left(\frac{2 \Delta G_{\mathrm{m}, \mathrm{a}}}{\mathrm{R} T_{\mathrm{a}}}\right)=\left(2-\frac{T_{2}}{T_{\mathrm{a}}}\right) \ln \left(\frac{k_{1} \mathrm{~h}}{\mathrm{k}_{\mathrm{B}} T_{1}}\right)+\left(2-\frac{T_{1}}{T_{\mathrm{a}}}\right) \ln \left(\frac{k_{2} \mathrm{~h}}{\mathrm{k}_{\mathrm{B}} T_{2}}\right)
\end{aligned}
$$


The operations to this point do not account for the transitional, cyclic nature of the issue and this feature is now accounted for by adjusting both In-terms for their geometric mean temperature $T_{\mathrm{g}}$ via the basic thermodynamic equilibrium relation $-R T \ln K=\Delta G_{m}{ }^{\circ}$ as equations $8 \mathrm{a}$ and $8 \mathrm{~b}$ show.

$$
\begin{aligned}
& -\left(\frac{\Delta G_{\mathrm{m}, x}^{\circ}}{\mathrm{R}}\right)=T_{x} \ln K_{\mathrm{x}}=T_{x} \ln \left(\frac{k_{x} \mathrm{~h}}{\mathrm{k}_{\mathrm{B}} T_{\mathrm{x}}}\right) \Rightarrow T_{x} \ln \left(\frac{k_{x} \mathrm{~h}}{\mathrm{k}_{\mathrm{B}} T_{\mathrm{x}}}\right)=T_{\mathrm{g}} \ln \left(\frac{k_{\mathrm{g}} \mathrm{h}}{\mathrm{k}_{\mathrm{B}} T_{\mathrm{g}}}\right) \Rightarrow \ln \left(\frac{k_{x} \mathrm{~h}}{\mathrm{k}_{\mathrm{B}} T_{\mathrm{x}}}\right)=\frac{T_{\mathrm{g}}}{T_{x}} \ln \left(\frac{k_{g} \mathrm{~h}}{\mathrm{k}_{\mathrm{B}} T_{\mathrm{g}}}\right) \\
& -\left(\frac{2 \Delta G_{\mathrm{m}, \mathrm{a}}^{\circ}}{\mathrm{R} T_{\mathrm{a}}}\right)=\ln \left(\frac{k_{g} \mathrm{~h}}{\mathrm{k}_{\mathrm{B}} T_{\mathrm{g}}}\right)\left(\left(2-\frac{2 T_{2}}{T_{1}+T_{2}}\right) \frac{T_{\mathrm{g}}}{T_{1}}+\left(2-\frac{2 T_{1}}{T_{1}+T_{2}}\right) \frac{T_{\mathrm{g}}}{T_{2}}\right)
\end{aligned}
$$

Combining equation $8 \mathrm{~b}$ with equation 4 yields equation 9 which is the baseline equation about the relation between macroscopic and microscopic level: so if only a relative $(y-z)$ margin of the battery capacity $(1 \geq y>z \geq 0)$ respective Gibbs enthalpy on the left is used what about the right?

$-(y-z)\left(\frac{2 \Delta G_{\mathrm{m}, \mathrm{a}}^{\circ}}{\mathrm{R} T_{\mathrm{a}}}\right)=\ln \left(\#_{\text {reaction events }}\right) ?$

The chemical potential of the ideal gas which is shown in equation 10 points a fundamental answer to that question: a difference of Gibbs enthalpies translates by principle in a logarithmic manner.

$\mu-\mu^{\circ}=\mathrm{R} T \ln \left(\frac{p}{p^{\circ}}\right)$

Yet how might that apply to equation 9? That a molecular image of battery capacity might clarify: the charging reaction will take the empty battery towards the capacity threshold $y$, which is a relative proportion of the specific total of reaction events. Then the system is discharged to a lower relative value $z$. So if the system starts in the charged state $y$, discharges to state $z$ and recharges back to state $y$ for a full cycle, the reaction event reservoir argument entails that the system is two times at the reaction event level of state $y$ and one time at those of state $z$. Hence a cycle between $(y, z)$-capacity margins has an intrinsic reference to $(2 y-z)$ regarding the filling level of the reaction even reservoir. Due to equation 10 respective the logarithmic nature of equation $9, \ln (2 y-z)$ is placed in lieu of the question mark as a denominator to $\ln$ (\# reaction events ) as shown in equation 11.

$-(y-z)\left(\frac{2 \Delta G_{m, a}{ }^{\circ}}{R T_{a}}\right)=\frac{\ln \left(\#_{\text {reaction events }}\right)}{\ln (2 y-z)}$ 
Accounting in some logarithmic manner for the relative capacity margins $y$ and $z$ gets a fundamental feature of the issue right as it makes a difference with regard to cycle-life which e.g. $40 \%$ of battery capacity are used: The (70/30) \% capacity margin yields a different cycle-life from those obtained from using (100/60) \% of battery capacity. The next adjustment is the substitution of the Gibbs enthalpy term in equation 11 according to equation $8 \mathrm{~b}$ and making the factor 2 in the temperaturerelated term on the left change place with the denominator on the right because by the model a fail of one reaction event causes the falling away of a complete cycle. That is shown in equation 12 and completes the meaning-wise transition from reaction event count towards cycle count.

$-(y-z) \ln \left(\frac{k_{\mathrm{g}} \mathrm{h}}{\mathrm{k}_{\mathrm{B}} T_{\mathrm{g}}}\right)\left(\left(1-\frac{T_{2}}{T_{1}+T_{2}}\right) \frac{T_{\mathrm{g}}}{T_{1}}+\left(1-\frac{T_{1}}{T_{1}+T_{2}}\right) \frac{T_{\mathrm{g}}}{T_{2}}\right) \ln (2 y-z)=\frac{\ln \left(\#_{\text {reaction events }}\right)}{2}=\ln \left(\#_{\text {cycles }}\right)$

The rate constant $k_{\mathrm{g}}$ in equation 12 is now expressed as the quotient of geometric mean specific power $P_{\mathrm{s}}$ by mean specific energy $E_{\mathrm{s}}$. Since the specific energy $E_{\mathrm{s}}$ of a battery cell is a quantity set by reaction stoichiometry, ${ }^{3}$ thus a setup-specific constant, the variable character of $k_{\mathrm{g}}$ rests with specific power $P_{\mathrm{s}}$ respective the mean $\sqrt{P_{1,5} P_{2, \mathrm{~s}}}$ of both reactions. Equation 13 shows this transformation for the common units of $\mathrm{W} \mathrm{kg}^{-1}$ respective $\mathrm{Wh} \mathrm{kg}^{-1}$ and pools all pre-logarithmic factors in a variable $\alpha$.

$-\alpha \ln \left(\frac{\sqrt{P_{1, \mathrm{~s}} P_{2, \mathrm{~s}}} \mathrm{~h}}{E_{\mathrm{s}} \mathrm{k}_{\mathrm{B}} \sqrt{T_{1} T_{2}}}\right)=\ln \left(\#_{\text {cycles }}\right)$

$E_{s}=\left[W h ~ k g^{-1}\right] \quad P_{x, s}=\left[\mathrm{Wkg}^{-1}\right] \quad \alpha=\ln (2 y-z) \cdot(y-z) \cdot\left(\left(1-\frac{T_{2}}{T_{1}+T_{2}}\right) \frac{T_{g}}{T_{1}}+\left(1-\frac{T_{1}}{T_{1}+T_{2}}\right) \frac{T_{g}}{T_{2}}\right)$ $1 \geq y>z \geq 0$

The arithmetic sign on the left is drawn into the In-term which is shown in equation 14.

$\alpha \ln \left(\frac{E_{\mathrm{s}}}{\sqrt{P_{1, \mathrm{~s}} P_{2, \mathrm{~s}}}} \frac{\mathrm{k}_{\mathrm{B}} \sqrt{T_{1} T_{2}}}{\mathrm{~h}}\right)=\ln \left(\#_{\text {cycles }}\right)$

$E_{s}=\left[W h k^{-1}\right] \quad P_{x, s}=\left[\mathrm{Wkg}^{-1}\right] \quad \alpha=\ln (2 y-z) \cdot(y-z) \cdot\left(\left(1-\frac{T_{2}}{T_{1}+T_{2}}\right) \frac{T_{g}}{T_{1}}+\left(1-\frac{T_{1}}{T_{1}+T_{2}}\right) \frac{T_{g}}{T_{2}}\right)$

$1 \geq y>z \geq 0$

It is discernible that equation 14 can be separated into a natural constant-temperature In-term and a setup-specific In-term: towards that end, time needs to be split among both by means of conversion factor $\tau=1 \mathrm{~h} / 3600 \mathrm{~s}$ for unit neutrality within both In-terms, which equation 15a shows (of the $\tau / \tau$ term the $\tau$ in the numerator is absorbed by $E_{s}$ at no further effect). The setup-specific term is then expressed by its negative reciprocal for its more instructive form which is shown in equation $15 \mathrm{~b}$. 


$$
\begin{aligned}
& \begin{aligned}
\ln \left(\#_{\text {cycles }}\right) & =\alpha \ln \left(\frac{E_{\mathrm{s}}}{\sqrt{P_{1, \mathrm{~s}} P_{2, \mathrm{~s}}}} \frac{\tau}{\tau} \frac{\mathrm{k}_{\mathrm{B}} \sqrt{T_{1} T_{2}}}{\mathrm{~h}}\right)=\alpha \ln \left(\frac{E_{\mathrm{s}} \tau}{\sqrt{P_{1, \mathrm{~s}} P_{2, \mathrm{~s}}}} \frac{3600[\text { seconds }] \mathrm{k}_{\mathrm{B}} \sqrt{T_{1} T_{2}}}{[1 \mathrm{~h}] \mathrm{h}}\right)+\alpha \ln \left(\frac{3600[\text { seconds }] \mathrm{k}_{\mathrm{B}} \sqrt{T_{1} T_{2}}}{\mathrm{~h}}\right) \\
& =\alpha \ln \left(\frac{E_{\mathrm{s}}}{\sqrt{P_{1, \mathrm{~s}} P_{2, \mathrm{~s}}}[1 \mathrm{hour}]}\right)
\end{aligned} \\
& \ln \left(\#_{\text {cycles }}\right)=\alpha \ln \left(\frac{3600[\text { seconds }] \mathrm{k}_{\mathrm{B}} \sqrt{T_{1} T_{2}}}{\mathrm{~h}}\right)-\alpha \ln \left(\frac{\sqrt{P_{1, \mathrm{~s}} P_{2, \mathrm{~s}}}[1 \mathrm{hour}]}{E_{\mathrm{s}}}\right) \\
& E_{\mathrm{s}}=\left[\mathrm{Wh} \mathrm{kg}{ }^{-1}\right] \\
& \tau=1 \mathrm{~h}(3600 \mathrm{~s})^{-1}
\end{aligned}
$$

A glance at the proportions between both In-terms in equations 15 suggests that a final regularity reminiscent of Carnot-efficiency underlies the entire matter of battery cycle-life! True to that notion, the battery-specific In-term is expressed under the premise of constant voltage by the geometric mean of $C$-rates to the charge and discharge process. These $C$-rate values, which may be data averages themselves, are either readily available information or can be estimated from application profiles or modelled on basis of reasonable generic values: Either way, equation 16 presents a most non-specific, ideal relation of general terms towards the maximum cycle-life of a secondary battery.

$$
\begin{aligned}
& \ln \left(\#_{\text {cycles }}\right)=\alpha \ln \left(\frac{3600\left[\text { seconds] } \mathrm{k}_{\mathrm{B}} \sqrt{T_{1} T_{2}}\right.}{\mathrm{h}}\right)-\alpha \ln \left(\sqrt{C \text {-rate } \text { charge }_{\text {-rate }} \text { discharge }}[1 \text { hour] })\right. \\
& \alpha=\ln (2 y-z) \cdot(y-z) \cdot\left(\left(1-\frac{T_{2}}{T_{1}+T_{2}}\right) \frac{T_{g}}{T_{1}}+\left(1-\frac{T_{1}}{T_{1}+T_{2}}\right) \frac{T_{g}}{T_{2}}\right) \quad 1 \geq y>z \geq 0
\end{aligned}
$$

\section{Discussion}

Equation 16 is the culmination point of an ideal approach and exactly because of that quality it has significance for real systems as a limit to the feasible. While the line of argument towards equation 16 is tight, it may be challenged whether intercalation of the migrating species into the vacant electrode site is indeed the highest activated, kinetically visible step of the reaction as this approach presupposes. It is not denied that this is a conception but it is a good one, resonating the principal direction in Li-battery research of aiming at easier Li-intercalation by opening up of the layers of e.g. graphite electrodes (e.g. by silicon or potassium). Another noteworthy aspect is that the model accounts for the influence of temperature by the deviation of the geometric from the arithmetic mean: for $T_{1}=T_{2}$ the temperature-term adopts the value of 1 , for $T_{1} \neq T_{2}$ it is always $<1$. The term $\mathrm{k}_{\mathrm{B}} \sqrt{T_{1} T_{2}}$ suggests a generally favourable effect of higher temperature(s) but it is to consider that degradation reactions, which also accelerate with temperature, are not part of the model.

The radical statement of equation 16 is that battery cycle-life is independent of battery-specific tangibles regarding energy and power in the strict, explicit sense: this solution comprises of two logarithmic terms of which the predominant one is defined by natural constants, temperature(s) and 
relative capacity margins; the argument of the other one comes down to the arithmetic mean of $C$ rates. Meaning-wise, the former term is reminiscent of Carnot-efficiency as it tells about the two energy levels of a cycle in terms of temperature(s) and relative capacity margins. The second term tells how fast the system is run through a cycle and both terms combine by the outlined minimum causality of failure to the figure how often that can be done at best: the ideal battery cycle-life.

In contrast to the current consensus opinion, which attributes the causality of changes to cycle-life in blanket fashion to the engineering of battery-specific tangibles, this work arrives at a much more differentiated picture: That changes to battery-specific tangibles may yield differences of several hundred or more cycles is here the consequence of them being reinforced by a long, natural constant-based, logarithmic lever. Due to the final exponential enhancement towards the cycle-life value, comparatively small changes taking effect at the tip of that long lever cause big differences. The approach is fundamental but may be refined further for better compliance with real systems (e.g. regarding failure mechanism) though that might not be necessary as engineering is capable of getting close to the ideal thermodynamic limits to reversible mass transfer - eventually. ${ }^{3}$

The findings of this work represent a fundamental answer and seemingly warrant nothing less than a revision of the current academic consensus on the matter. That however might mean asking for (too?) much because the mere existence of a solution of the kind is considered an impossibility by the empiric positivist bias dominating the field of reversible chemical energy storage ever since: Yet the notion of battery cycle-life being virtually or exclusively a matter of empiric nature is not sound to begin with as suggesting that the nature of a material item could be studied solely by methods based on the principles of causality to matter while denying the possible significance or even existence of any higher regularity ensuing from them. That is contradictory, either there are general principles to natural science or there are none, which is of course not a matter open to choice.

Hence discussing this work's result in terms of that incomplete yet authorative position and the results it bore forth is insofar pointless as this outcome originates from diametrically opposed premises and it is only consequential that its qualities, namely convergence in outcome, general significance and timeless validity reflect that. Thus, the only possible common ground might be the factual level of cycle-life figures. The difference is principal and has a long history, ultimately stretching back to the dualism between Aristotle and Plato as far as Western thinking is concerned.

Even though this divergence about the philosophy of science is a matter vital to this work and would thus merit a wider discussion, this is something beyond the means of this paper. A dedicated effort another day may address this point with due depth towards a balanced, abiding dialectic resolution.

As far as the factual dimension of reversible chemical energy storage is concerned, the befogging empiric positivist monopoly of opinion has been broken and those who wish to see may now see.

\section{Conclusion}

An approach based on physical chemistry fundamental on the issue of maximum battery cycle-life leads to an ideal model of general validity: The result can be understood further in the sense of a thermodynamic final regularity similar to Carnot-efficiency which challenges the hitherto academic consensus opinion that battery cycle-life were an issue of virtually or exclusively empiric nature. 


\section{Acknowledgements}

This paper spun-off from this author's prime line of work done at FOTEC Forschungs- und Technologietransfer GmbH under European Space Agency grant 4000105330/12/NL/CLP and European Defence Agency grant A-1341-RT-GP which is all gratefully acknowledged.

\section{Conflicts of Interest}

There are no conflicts to declare.

\section{References}

(1) Motapon, S. N.; Lachance, E.; Dessaint, L.-A.; Al-Haddad, K. A Generic Cycle Life Model for Lithium-lon Batteries Based on Fatigue Theory and Equivalent Cycle Counting. IEEE Open J. Ind. Electron. Soc. 2020, 1, $207-217$. https://doi.org/10.1109/OJIES.2020.3015396.

(2) Pawelke, R. H. The Master Key to the Problem of Reversible Chemical Hydrogen Storage Is $12 \mathrm{KJ}\left(\mathrm{Mol} \mathrm{H}_{2}\right)^{-1}$; preprint; 2021. https://doi.org/10.26434/chemrxiv.6940379.v10.

(3) Pawelke, R. H. The Straightforward Route Towards the Theoretical Specific Free Enthalpy of Electrochemical Reactions; preprint; 2021. https://doi.org/10.26434/chemrxiv.8799188.v3.

(4) Ptáček, P.; Šoukal, F.; Opravil, T. Introduction to the Transition State Theory. In Introducing the Effective Mass of Activated Complex and the Discussion on the Wave Function of this Instanton; Ptáček, P., Opravil, T., Šoukal, F., Eds.; InTech, 2018. https://doi.org/10.5772/intechopen.78705. 